\title{
A patient with complete androgen insensitivity syndrome (CAIS) presenting as a case of indirect inguinal hernia: a case report
}

Intruduction:

$$
\text { A El Mirghani }{ }^{1} \text { FRCSI, A M Musa }{ }^{2} \text { FRCSI }
$$

The management of intersex patients is a challenge. Although in the majority of patients the diagnosis may be made on the basis of cytogenetic and biochemical tests, there is a selective group of patients with difficulties in the establishment of final diagnosis and gender assignment ${ }^{1}$.

Complete androgen insensitivity syndrome, commonly known as the testicular feminization syndrome, is characterized by a 46, XY karyotype, bilateral testes, absent or hypoplastic Wolffian ducts, and female-appearing external genitalia with diminished axillary and pubic hair development. Although initial diagnosis in the child is difficult, the syndrome must be suspected after puberty if primary amenorrhea is present ${ }^{2}$. Androgen insensitivity syndrome (AIS) is an X-linked recessive disorder characterized by impairment of the androgen-dependant male sexual differentiation ${ }^{3}$.

\section{Case report}

A 28-year old female presented with a swelling in the right groin for 13 years. She experienced pain in the last few weeks. The swelling disappears when she lies down and appears and increases in size with her daily activities. She is married for 4 years but has no children. She didn't experience menarche (primary amenorrhoea).

On physical examination she looked well, not pale jaundiced or cyanosed. Has well developed breasts (Fig 1). Chest and cardiovascular system were normal. Abdomen showed sparse pubic hair. There was a right groin swelling which was above and medial to the pubic tubercle, reducible and has a cough impulse. The left groin was normal. Local examination revealed normal female external genitalia. She was diagnosed as a case of right sided indirect inguinal hernia.

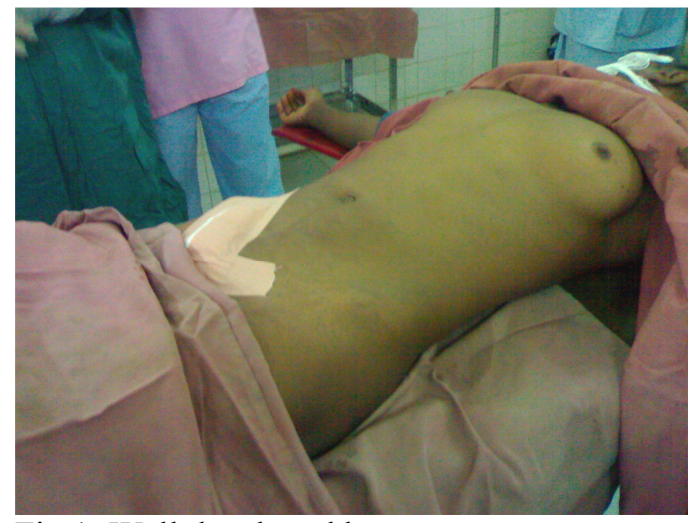

Fig 1: Well developed breasts

At operation a sac of an indirect inguinal hernia was found. The sac was opened

1. Associate Prof. Head department of Surgery.

2. 2. Associate Prof. Department of

Surgery.Omdurman Islamic University. and a well developed testis with a normal epididymis and spermatic cord was found. Right sided orchidectomy was done (Fig 2).

Subsequently, histopathology reported immature testis. The tubules were lined with Sertoli cell sand spermatogonia only. There was some interstitial fibrosis and Leydig cell hyperplasia. The tunica vaginalis was thick. The epididymis was rudimentary and cystic.

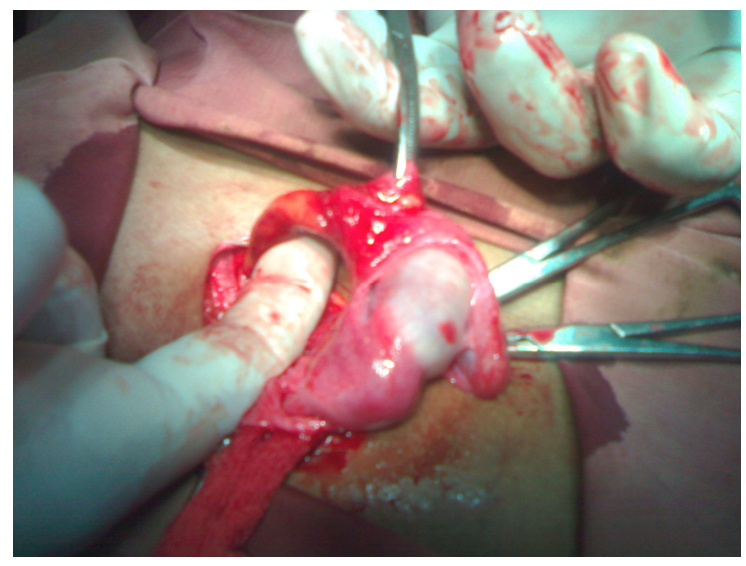

Fig 2: Right inguinal hernial sac containing externally well developed testis

Buccal mucosal smear for Barr bodies was negative. CT scan of the abdomen and pelvis revealed normal intra abdominal and pelvic organs (Fig 3), uterine cavity could not be seen, however small uterus like structure was seen and so was the vagina.

Diagnostic laparoscopy showed an empty pouch of Douglas but testicle on the left side was found. Laparoscopic left orchidectomy was done. 


\section{Discussion}

The androgen receptor (AR) is a member of the superfamily of nuclear receptors that function as ligand-dependent transcription factors. The AR gene lies on the X-chromosome at Xq1112. Like other nuclear receptors, AR contains four domains, the N-terminal domain (NTD), the DNA-binding domain (DBD), the hinge region and the ligand- or androgen-binding domain (LBD). The DBD and the LBD show considerable homology to other nuclear receptors. The DBD contains two zinc fingers and is required for androgen response element recognition. The 253 residue C-terminal LBD contains $12 \alpha$-helices and the highly hydrophobic ligand-binding site. Mutations in the AR are associated with variety of diseases. Androgen insensitivity syndrome (AIS) is associated with germline mutations within the AR. AIS is classed into three clinical phenotypes, complete (CAIS), partial (PAIS) and mild (MAIS) insensitivity. CAIS patients exhibit a male genotype with female external genitalia. PAIS patients exhibit a wide range of phenotypes, often with ambiguous external genitalia. MAIS patients are sterile and have male external genitalia. Mutations for each of these phenotypes are spread throughout the AR, with most mutations occurring in the LBD in the C-terminal end of the receptor. Mutation results in the replacement of a highly conserved leucine residue with phenylalanine in ligand-binding domain of the receptor located in helix 10 of the androgen receptor ${ }^{4}$.

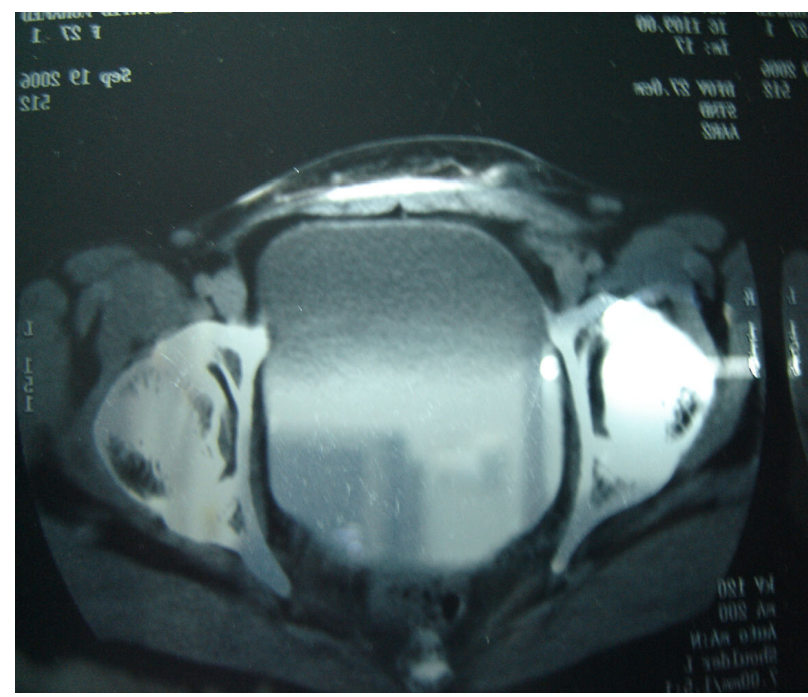

Fig 3: CT showing normal pelvic organs but uterus was not seen

Development of the male genitalia is largely controlled by cells in the urogenital mesenchyme that express androgen receptors (AR). In general, the degree of genital ambiguity correlates with the level of compromise of AR function: 46, XY individuals with AR-inactivating mutations are completely feminized despite high levels of serum testosterone. The molecular events responsible for AR-dependent male genital morphogenesis are poorly understood. Although there are striking differences in the gene expression profiles of genital fibroblasts cultured from normal and AIS patients, but no transcriptional response to androgen was detectable in any of the cultured genital fibroblasts for the eventual phenotype in the patients ${ }^{5}$. Histology of patient's gonads showed Leydig cell hyperplasia, with few or no spermatogonia. It is thought that $\mathrm{AR}$ gene mutations result in hormonal imbalance, resulting in the high levels of luteinizing hormone (LH) and ultimately Leydig cell hyperplasia or tumor formation.

Patients with androgen insensitivity syndrome show predisposition to germ cell tumours in the adult testis. These tumours may originate from an in situ carcinoma that might have been present in childhood. Testicular biopsy is warranted as early as the syndrome is diagnosed, but orchidectomy because of the risk of malignancy is not advised until after puberty or if carcinoma in situ is diagnosed when orchidectomy is indicated. As the chance of diagnosing carcinoma in situ from a single biopsy in a prepubertal testis is unknown, a negative finding in childhood should be confirmed by another biopsy at puberty if orchidectomy is not to be performed ${ }^{6}$.

In Germany, an incidence of 2 per 10,000 births with ambiguous genitalia per year was found. Prevalence was higher in infants from nonGerman family background ${ }^{7}$. In more than $50 \%$ of all infants a definite diagnosis was lacking even at the age of 6 months. In those cases where the etiology was confirmed, the most common diagnosis was congenital adrenal hyperplasia, followed by androgen insensitivity syndrome and mixed gonadal dysgenesis. In contrast an epidemiological study not only in maternity hospitals and nurseries but in adult units in Sudan is needed to for decided the incidence of ambiguous genitalia and AIS in Sudan ${ }^{7}$.

Disclosing the fact that the patient is genotypically a male can be emotionally harmful to a patient who is leading a lifestyle of a normal female, yet, the patient should know that her syndrome does not worsened by time but is uncorrectable. The help that can be provided 
include gonadectomy because of the risk of malignancy, surgical reconstruction of the vagina and counseling on adoption. They should be referred to as females as they are displaying the psychological and physical characters of females8. The physician prime duty is to protect his patients from harmful physical and psychological insults.

\section{References:}

1.Chertin B, Koulikov D, Alberton J et al. The use of laparoscopy in intersex patients. Pediatr Surg Int. 2006 May;22(5):405-8

2. Tokgoz H, Turksoy $\mathrm{O}$, Boyacigil $\mathrm{S}$ et al. Complete androgen insensitivity syndrome: report of a case with solitary pelvic kidney. Acta Radiol. 2006 Mar;47(2):222-5

3. Tokgoz H, Turksoy $\mathrm{O}$, Boyacigil $\mathrm{S}$ et al. Complete androgen insensitivity syndrome: report of a case with solitary pelvic kidney. Acta Radiol. 2006 Mar;47(2):222-5

4. Mooney SD, Klein TE, Altman RB et al. A functional analysis of disease-associated mutations in the androgen receptor gene. Nucleic Acids Res. 2003; 31(8): 42.

5. Mooney SD, Klein TE, Altman RB et al. A functional analysis of disease-associated mutations in the androgen receptor gene. Nucleic Acids Res. 2003; 31(8): 42.

6. Muller J, Skakkebaek NK. Testicular carcinoma in situ in children with the androgen insensitivity (testicular feminization) syndrome. B M J 1984 (288): 1419-1420

7. Thyen U, Lanz K, Holterhus PM. et al. Epidemiology and initial management of ambiguous genitalia at birth in Germany. Horm Res. 2006;66(4):195-203

8. Natarajan A. Medical ethics and truth telling in the case of androgen insensitivity syndrome. $\mathrm{Ca}$ Med Assoc J. 1996; 154 (4): 568-70 